21st Particles and Nuclei International Conference (PANIC 2017)

International Journal of Modern Physics: Conference Series

Vol. 46 (2018) 1860069 (7 pages)

(C) The Author(s)

DOI: $10.1142 / \mathrm{S} 2010194518600698$

\title{
Measurements of Properties of Antihydrogen
}

\author{
Art Olin (for the ALPHA Collaboration) \\ TRIUMF/University of Victoria, 4004 Wesbrook Mall \\ Vancouver, BC, V6J2B3, Canada \\ olin@triumf.ca
}

\begin{abstract}
M. Ahmadi, B.X.R. Alves, C.J. Baker, W. Bertsche, E. Butler, A. Capra, C. Carruth, A.E. Charman, L.T. Evans, C.L. Cesar, M. Charlton, S. Cohen, R. Collister, S. Eriksson, A. Evans, L.T. Evans, N. Evetts, J. Fajans, T. Friesen, M.C. Fujiwara, D.R. Gill, J.S. Hangst, W.N. Hardy, M.E. Hayden, C.A. Isaac, M.A. Johnson, J.M Jones, S.A. Jones, S. Jonsell, A. Khramov, P. Knapp, L. Kurchaninov, N. Madsen, D. Maxwell, J.T.K. McKenna, S.Menary, T. Momose, J.J. Munich, K. Olchanski, A. Olin, P. Pusa, C. Rasmussen, F. Robicheaux, R.L. Sacramento, M. Sameed, E. Sarid, D.M. Silveira, G. Stutter, C. So, T.D. Tharp, R.I. Thompson, D.P. van der Werf, J.S. Wurtele, A.I. Zhmoginov
\end{abstract}

Published 3 May 2018

\begin{abstract}
The ALPHA project at the CERN AD is testing fundamental symmetries between matter and antimatter using trapped antihydrogen atoms. The spectrum of the antihydrogen atom may be compared to ordinary hydrogen where it has been measured very precisely. CPT conservation, which underpins our current theoretical framework, requires equality of the masses and charges of matter and its antimatter partners, so antihydrogen spectroscopy presents a path to precision CPT tests.

I will discuss the techniques used by ALPHA to trap more than 8000 antihydrogen atoms in 2016, and interrogate them for $600 \mathrm{~s}$. The $1 \mathrm{~S}-2 \mathrm{~S}$ transition in antihydrogen has been observed for the first time, and it agrees with its hydrogen counterpart within an uncertainty of $400 \mathrm{kHz}$ or $0.2 \mathrm{ppb}$. The charge of the antihydrogen atom has been bounded below $0.710^{-9} e$. A value of $1420.40 .5 \mathrm{MHz}$ for the hyperfine splitting has been obtained from observation of the positron spin resonance spectrum.
\end{abstract}

Keywords: Antimatter; antihydrogen; matter/antimatter symmetry.

\section{Introduction}

As a consequence of his formulation of a relativistic quantum mechanics, Paul Dirac predicted the existence of antimatter, unseen counterparts to the spin 1/2 particles with opposite charge but with otherwise identical properties ${ }^{1}$. This prediction was met with great scepticism. Experimental verification came swiftly with discovery

This is an Open Access article published by World Scientific Publishing Company. It is distributed under the terms of the Creative Commons Attribution 4.0 (CC-BY) License. Further distribution of this work is permitted, provided the original work is properly cited. 


\section{A. Olin}

of the positron by Carl Anderson ${ }^{2}$, exciting great public interest. Physical theories were assumed at that time to satisfy not only Lorentz invariance, the continuous symmetry of special relativity, but also its finite symmetries of parity $(\mathrm{P})$, time reversal $(\mathrm{T})$, and charge conjugation $(\mathrm{C})$. In this context charge conjugation was recognized as the symmetry between matter and antimatter. While it has been experimentally determined that the $\mathrm{P}$ and $\mathrm{CP}$ symmetries are broken in weak processes, there has been no observed violation of the combination CPT. It has been shown ${ }^{3}$ that quantum field theories satisfying locality and causality and having a hermitian lagrangian must conserve CPT, so any violation would fundamentally impact our present theoretical framework. CPT invariance predicts the equality of the energy levels and decay widths in hydrogen and antihydrogen, and that the charges of matter particles and their corresponding antiparticles are equal in magnitude - this is also a consequence of gauge invariance. Our experimental program is to compare properties of hydrogen $(H)$ and antihydrogen $(\bar{H})$ with the highest possible precision. The corresponding measurements in $H$ are among the most accurate in experimental physics. Other talks in this session have explored the consequences of breaking CPT in more detail. This talk will cover only the CPT tests performed by the ALPHA collaboration.

\section{Making and Trapping Antihydrogen}

The measurements I will describe involve synthesizing antihydrogen from antiprotons $(\bar{p})$ from the CERN AD, and positrons $(\mathrm{e}+)$ from our accumulator, in a Penning trap, catching some of them in a magnetic trap, and then interrogating them to study the observable of interest. The trap ${ }^{4}$, schematically shown in Fig 1 is surrounded by a silicon vertex detector (SVD) which efficiently detects the time and position of $\bar{p}$ or $\bar{H}$ annihilation vertices of on the trap electrodes. This can be detected during the interrogation, and the number surviving interrogation can also be measured by counting annihilations when the trap magnets are de-energized. We

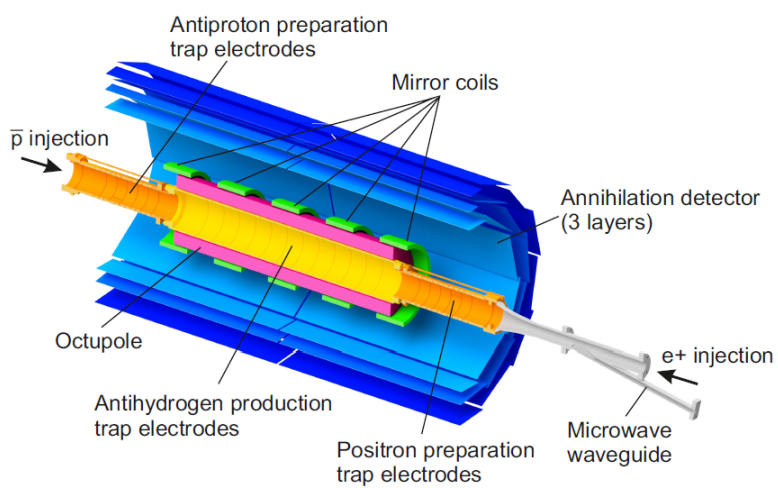

Fig. 1. Schematic of ALPHA2 atom trap. Not shown is a solenoid magnet surrounding the apparatus and producing a $1 \mathrm{~T}$ field. 
trapped our first antihydrogen in 2010, achieving a rate of 0.2 observed annihilation vertices / trial ${ }^{5}$. Currently $90000 \bar{p}$ and $1.610^{6} e^{+}$are mixed, producing 25000 $\bar{H}$, most of which are too energetic to trap. The improvements enabling our present 25 trapped $\bar{H}$ /trial are: a new cryostat providing a stable thermal environment, rotating wall compression in the strong drive regime coupled with evaporative cooling to produce highly reproducible e + and $\bar{p}$ plasmas ${ }^{6}$, slow merging of the e+ and $\bar{p}$ plasmas to synthesize $\bar{H}$, and stacking of multiple $\bar{H}$-synthesis cycles in the atom $\operatorname{trap}^{7}$ for a single interrogation.

\section{Limit on $\bar{H}$ charge from Stochastic Acceleration}

The $\bar{H}$ atom offers us the ability to measure directly a small difference between the charges of its components, which is much easier than measuring the components directly. In this experiment ${ }^{8}$ the charge is probed by applying stochastically varying potentials to the Penning electrodes, subjecting the trapped $\bar{H}^{\prime} s$ to random $100 \mathrm{~V}$ kicks. Over two days in 2015 we performed 10 runs with stochastic potentials applied during a 120 s hold period, and observed $12 \bar{H}$ annihilations when the trap magnets were de-energized at the end of the hold. These were interleaved with 10 runs having the same hold period but no applied potentials. $12 \bar{H}$ annihilations were also observed at the end of these runs, so there is no evidence that the potentials expelled $\bar{H}^{\prime} s$ from the trap.

A simple estimate of the expected energy gain of a charge $Q e$ at the end of the hold period after $\mathrm{N} \Delta \Phi$ volt kicks is $\sqrt{N} \Delta \Phi|Q| e$. We used $\Delta \Phi=100 \mathrm{~V}$ and $N=$ 84900 kicks, and a trap depth of $0.5 \mathrm{~K}$ yielding an estimate of $|Q|<1.610^{-9}$. A value of the survival probability is obtained from simulating trajectories using a detailed model of our trap and the applied stochastic potentials for a range of $\mathrm{Q}$ values. A Bayesian determination of the range of survival probabilities corresponding to a $1 \sigma$ variation in the data yields a bound of $|Q|<0.710^{-9}$. This includes a systematic uncertainty for the trap model. An order-of-magnitude improvement in this bound could be obtained by increasing the hold time and using stacking and higher trapping rates.

Our bound may be used (Fig 2) together with the precise measurements of $\bar{p} H e$ atomic transitions ${ }^{9}$, the $\bar{p}$ cyclotron frequency ${ }^{10}$, and the $1 \mathrm{~S}-2 \mathrm{~S}$ transition in positronium $^{11}$ to put a $1 \sigma$ bound of $0.710^{-9}$ on a positron charge anomaly - a factor 40 improvement. The bound on a positron mass anomaly $\frac{m_{e^{-}}-m_{e^{+}}}{m_{e^{-}}}$is also improved to $2010^{-9}$.

\section{Measurement of the $\bar{H}$ Hyperfine Splitting}

Fig 3 shows the expected energy levels of ground state antihydrogen in a magnetic field. Only the $\mid c>$ and $\mid d>$ levels are trapped, and driving the $|c>\rightarrow| b>$ and $|d>\rightarrow| a>$ transitions with microwave radiation expels the $\bar{H}$ from the trap. Our 2012 proof-of-principle measurement of these $\bar{H}$ positron spin resonances (PSR $)^{12}$ 


\section{A. Olin}

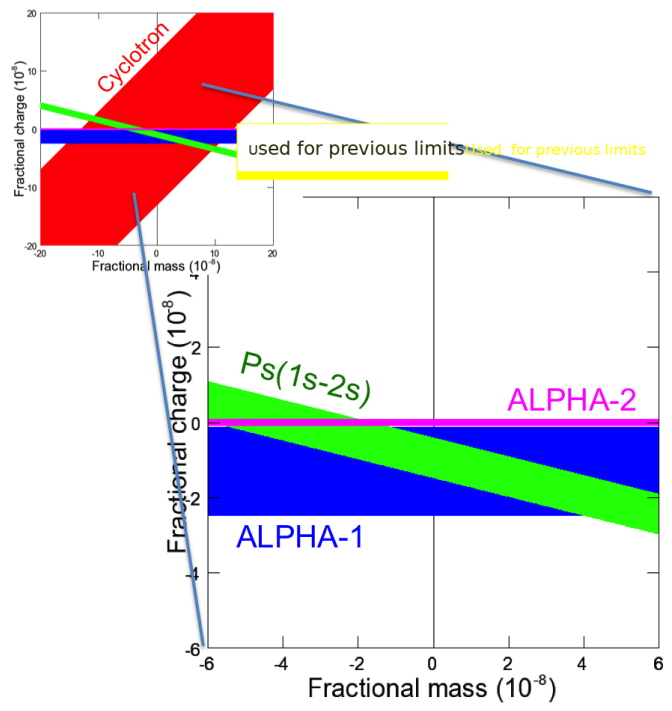

Fig. 2. Bounds on the positron mass and charge anomalies. Previous bounds came from the overlap of cylotron frequency and positronium 1S-2S measurements.

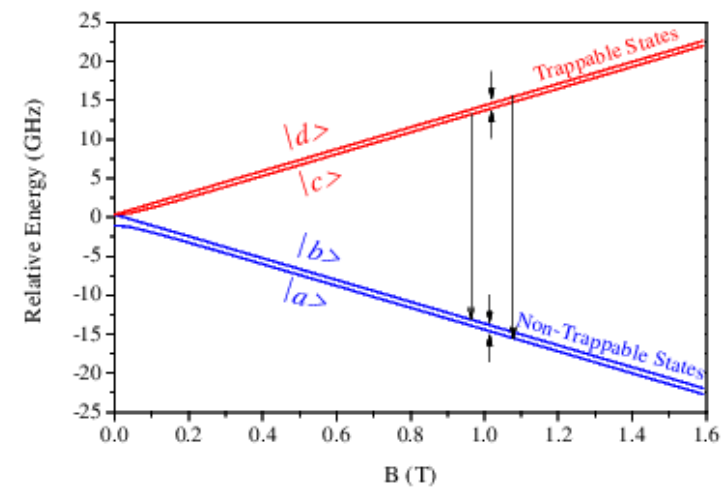

Fig. 3. Ground state antihydrogen energy levels in a magnetic field. The ALPHA field is 1T, and we drive the $|c>\rightarrow| b>$ and $|d>\rightarrow| a>$ transitions.

with $100 \mathrm{MHz}$ precision was limited by our knowledge and control of the magnetic fields in our apparatus. The improvements in our ability to measure and control the fields, together with the increased trapping rate allowing measurement of both transitions in a single trial has enabled us to measure the lineshapes of these resonances in the same field, subject only to short-term drifts ${ }^{13}$. The inhomogeneous trap field produces an asymmetric lineshape with a sharply defined onset at the field minimum. The frequency difference between the two onsets represents the ground state hyperfine splitting is independent of the field strength and the number of anti-atoms trapped. The field at trap center measured daily to $0.3 \mathrm{mT}$ from electron 


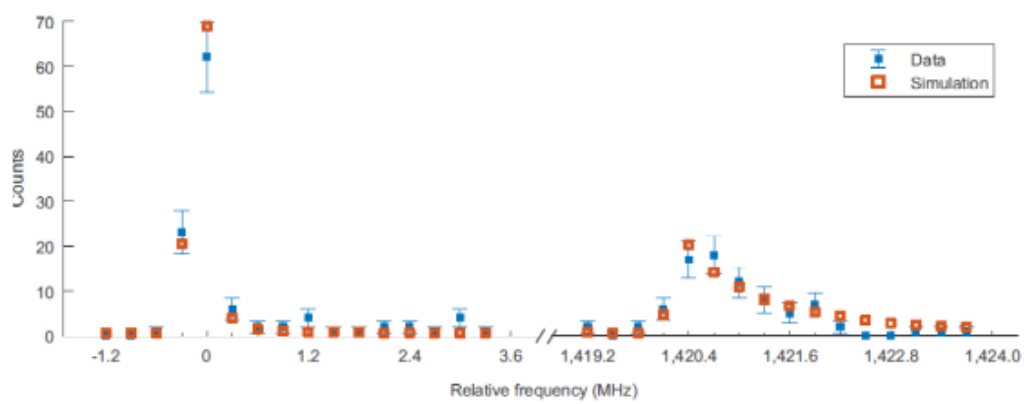

Fig. 4. Spectrum of the $|c>\rightarrow| b>/$ (lower) and $|d>\rightarrow| a>$ ground state transitions.

cyclotron resonance measurements on small electron plasmas. This also determines the microwave electric field strength at the trap center.

The run protocol consisted of irradiating 14 trapped $\bar{H}$ s over the $|c>\rightarrow| b>$ transition in $16300 \mathrm{kHz}$ steps advancing every $4 \mathrm{~s}$ with $160 \mathrm{~mW}$ microwaves, scanning the $|d>\rightarrow| a>$ transition at the same rate with $320 \mathrm{~mW}$ microwaves, and de-energizing the trap to detect the survivng $\bar{H}$ s. The results of 22 such runs taken over 3 days is shown in Fig 4. dependence of the microwave transmission through the electrode column. The lineshape is determined by the spin-flip probability and the number and trajectories of the remaining $\bar{H}$. Good agreement with a simulation using the measured microwave power and the trap dynamics can be seen. The difference in shape of the upper and lower transitions comes from the frequency dependence of transmitted microwave power. The resulting hyperfine splitting is $a / h=1,420.4 \pm 0.5 \mathrm{MHz}$, consistent with expectations for atomic hydrogen at the level of four parts in $10^{4}$. There are excellent prospects for another order-ofmagnitude improvement by using finer bins and better categorization of field drifts. A direct measurement of the $|d>\rightarrow| c>$ transition is potentially more precise, but radiation at that frequency is not transmitted by the electrode column. Work is proceeding on a resonator/electrode for this measurement. A complementary zero-field measurement is being pursued by the Asakusa Collaboration ${ }^{14}$.

\section{Observation of the $\bar{H}$ 1S-2S Transition}

The 1S-2S transition in hydrogen has been measured to an astounding few parts in $10^{-1515}$. Measuring it in antihydrogen to test CPT conservation was the original motivation for the ATRAP and ATHENA/ALPHA experiments. Performing this measurement on small numbers of trapped antihydrogen atoms instead of a cold atomic beam introduces additional challenges ${ }^{16}$.

The ALPHA2 cryostat has access ports for four laser ports, each crossing the trap center at an angle of $2^{\circ}$. A particular challenge is the Fabry-Pérot buildup cavity installed within the UHV volume of the cryostat. the alignment piezzo must have sufficient adjustment under these conditions. A cavity finesse of 250 and power $>1 W$ with a linewidth $<10 k H z$ has been achieved. 


\section{A. Olin}

The transitions of interest will excite the trapped $1 S \mid c>$ and $\mid d>$ levels (Fig 3) to their corresponding $2 \mathrm{~S}$ levels. The frequencies differ primarily because the hyperfine splitting of the $1 \mathrm{~S}$ and $2 \mathrm{~S}$ states are different. The trapping field is flattened using the inner three mirror coils to maximize the volume of resonance overlap with the laser beam. An $\bar{H}$ in the $2 \mathrm{~S}$ state can be ionized by another $243 \mathrm{~nm}$ photon, decay back to its original state, or decay via $2 \mathrm{P}$ mixing to a $1 S \mid a>$ or $\mid b>$ state which escapes the trap and annihilates. An experimental run consisted of irradiating 10 trapped $\bar{H}$ s at the $1 S|d>\rightarrow 2 S| d>$ hydrogen transition frequency for $300 \mathrm{~s}$, repeating this for the $1 S|c>\rightarrow 2 S| c>$ frequency, and de-energizing the trap. These were interleaved with runs with their frequencies detuned by $200 \mathrm{kHz}$, and with runs with the same hold times but no laser.

Table 1. 1S-2S Resonance Yields

\begin{tabular}{lccccc}
\hline Series Type & $\begin{array}{c}\text { Surviving } \\
\text { Counts }\end{array}$ & $\begin{array}{c}\text { Lost } \\
\%\end{array}$ & $\begin{array}{c}\text { Appearance } \\
\text { Counts }\end{array}$ & $\begin{array}{c}\text { Appearance } \\
\%\end{array}$ & $\begin{array}{c}\text { Power } \\
\text { mW }\end{array}$ \\
\hline Off Resonance & 159 & $56 \pm 7$ & 7 & $71 \pm 16$ & 1100 \\
On Resonance & 67 & & 79 & & 1100 \\
No Laser & 142 & $-16 \pm 6$ & 5 & $4 \pm-8$ & 0 \\
\hline
\end{tabular}

Consistent resonance signals are clearly seen both during and following the laser irradiation. Within the $400 \mathrm{kHz} @ 121 \mathrm{~nm}$ bound the $H$ and $\bar{H}$ transitions have the same energy. A measurement of the lineshape with a precision goal of several ppt is currently being attempted.

\section{Summary}

Measurements of the $\bar{H}$ charge, hyperfine splitting and 1S-2S transition have placed more stringent limits on a difference between $H$ and $\bar{H}$, and significantly improvement in these values is expected in the near future.

\section{Acknowledgments}

Financially supported by: the European Research Council through its Advanced Grant programme (JSH); CNPq, FAPERJ, RENAFAE (Brazil); NSERC, NRC/TRIUMF, EHPDS/EHDRS, FQRNT (Canada); FNU (NICE Centre), Carlsberg Foundation (Denmark); JSPS Postdoctoral Fellowships for Research Abroad (Japan); ISF (Israel); STFC, EPSRC, the Royal Society and the Leverhulme Trust (UK); DOE, NSF (USA); and VR (Sweden).

\section{References}

1. P.A.M. Dirac, Proceedings of the Royal Society A 117 610(1928).

2. C.D. Anderson, Phys. Rev. 43, 491, (1933). 
3. G. Lüders, Kongelige Danske Videnskabernes Selskab, Matematisk-Fysiske Meddelelser 28, 1(1954). See also J. Schwinger, Phys. Rev. 82,914(1951).

4. ALPHA Collaboration (C. Amole et al.Nucl. Instrum. Methods in Phys. Res. A 735,319(2014).

5. ALPHA Collaboration (C. Amole et al). Nature 468 673(2010).

6. ALPHA Collaboration (M. Ahmadi et al). Enhanced Control and Reproducibility of Nonneutral Plasmas to appear in Phys. Rev. Lett.

7. ALPHA Collaboration (M. Ahmadi et al). Nature Comm 8, 681(2017).

8. ALPHA Collaboration (M. Ahmadi et al). Nature $529373(2016)$.

9. M. Hori et al. Science 354610 (2016).

10. S. Ulmer et al. Nature $\mathbf{5 2 4} 196$ (2015).

11. M.S Fee et al. Phys. Rev. A 48 192(1993).

12. ALPHA Collaboration (C. Amole et al). Nature 483 439(2012).

13. ALPHA Collaboration (M. Ahmadi et al). Nature 548 66(2017).

14. N. Kuroda et al.JPS Conf. Proc. 18 011009(2017).

15. C.G. Parthey, et al. Phys. Rev. Lett. 107, 203001 (2011).

16. ALPHA Collaboration (M. Ahmadi et al). Nature 541 506(2017). 